\title{
Rivaroxaban: A New Treatment Paradigm in the Setting of Vascular Protection?
}

\author{
Rupert Bauersachs ${ }^{1}$ Faiez Zannad ${ }^{2}$ \\ ${ }^{1}$ Department of Vascular Medicine, Klinikum Darmstadt GmbH, \\ Darmstadt, Germany \\ ${ }^{2}$ Clinical Investigation Center, Centre Hospitalier Universitaire de \\ Nancy, Nancy, France
}

\begin{abstract}
Address for correspondence Rupert Bauersachs, Department of Vascular Medicine, Klinikum Darmstadt GmbH, Darmstadt, Germany (e-mail: rupert.bauersachs@mail.klinikum-darmstadt.de).
\end{abstract}

\begin{abstract}
Keywords

- coronary artery disease

- heart failure

- peripheral arterial disease

- rivaroxaban

- thrombosis

The pathophysiology of atherosclerosis involves a diseased endothelium, lipid accumulation and low-grade inflammation. In later stages of coronary artery disease (CAD) and peripheral arterial disease (PAD), plaque rupture may induce atherothrombosis caused by fibrin formation and platelet activation, leading to vessel occlusion with subsequent organ damage such as myocardial infarction, stroke or limb ischaemia. Because of the high disease burden associated with CAD and PAD, there is a need for continuous vascular protection beyond currently available treatments including antiplatelet agents. Due to its central role in the coagulation cascade, inhibition of factor $\mathrm{Xa}$, with the subsequent reduced thrombin formation that impacts not only fibrin but also platelets, may provide additional benefit over using antiplatelets alone. Evidence from Anti-Xa Therapy to Lower Cardiovascular Events in Addition to Standard Therapy in Subjects with Acute Coronary Syndrome-Thrombolysis In Myocardial Infarction-51 (ATLAS-ACS 2-TIMI 51) supports the use of the direct, oral, factor Xa inhibitor rivaroxaban ( $2.5 \mathrm{mg}$ twice-daily [bid] and $5 \mathrm{mg}$ bid) in reducing mortality and morbidity in patients with acute coronary syndrome, when combined with antiplatelets. Here, we review the role of rivaroxaban in three clinical trials of CAD and/or PAD: Cardiovascular OutcoMes for People using Anticoagulation StrategieS (COMPASS), Vascular Outcomes studY of ASA alonG with rivaroxaban in Endovascular or surgical limb Revascularization for PAD (VOYAGER PAD) and Cardiovascular Outcome Modification, Measurement AND Evaluation of Rivaroxaban in patients with Heart Failure (COMMANDER HF).
\end{abstract}

\section{Introduction}

Coronary artery disease (CAD) includes the clinical consequences of coronary artery atherothrombosis, including angina pectoris and myocardial infarction (MI). CAD is the most common single cause of death worldwide. ${ }^{1,2}$ In 2015 , the Global Burden of Disease collaboration highlighted the increasing incidence of mortality from the effects of CAD, due to the increasing age of the global population. ${ }^{3}$ The
American Heart Association (AHA) updated its Heart Disease and Stroke Statistics in 2017, which showed that 16.5 million U.S. adults aged $\geq 20$ years have CAD and its prevalence increases with age in women and in men. ${ }^{4}$

In 2013, a total of 202 million people were estimated to be living with peripheral arterial disease (PAD) and the prevalence of PAD was approximately $5 \%$ in patients aged 45 to 49 years, rising to approximately $18 \%$ in those aged 85 to 89 years. ${ }^{5}$ PAD is an atherosclerotic process that leads to received

September 29, 2017

accepted after revision

January 15, 2018
Copyright @ 2018 Schattauer
DOI https://doi.org/ 10.1055/s-0038-1636530. ISSN 0340-6245. 
stenosis and occlusion of non-cerebral and non-coronary arteries. These are typically arteries of the lower extremities -the resulting limb ischaemia can lead to amputation. ${ }^{6}$ PAD of the lower extremity is recognized as the third leading cause of atherosclerotic cardiovascular (CV) morbidity, after CAD and stroke. ${ }^{5,7}$ The risk of CV death is increased 5-fold in patients with asymptomatic PAD and 11 -fold in patients with symptomatic PAD, compared with patients without PAD. ${ }^{8}$

Evidence surrounding the real-world risk and clinical consequences of atherothrombosis is also available. The REACH registry is a global observational study of around 68,000 patients in 44 countries who are at high risk of atherothrombosis. ${ }^{9}$ Findings from the REACH registry show that a significant proportion (15.9\%) of the symptomatic population has polyvascular disease, encompassing CAD, PAD and/or cerebrovascular disease, and it was generally concluded that, despite the associated risks, $\mathrm{CV}$ disease is not being optimally managed. ${ }^{9}$ Patients with CAD and/or PAD have an unmet need for effective prevention of atherothrombotic events. The risk of atherothrombotic events in these patients remains high, as often aggressive antiplateletonly treatment has not conferred sufficient protection benefits. ${ }^{10-13}$

Atherothrombosis, induced by ruptured atherosclerotic plaques, occupies a central role in the pathogenesis of CAD and PAD. Rupture of the plaque is thought to be similar to acute coronary syndrome (ACS) in pathogenic terms, and this event causes the thrombogenic subendothelial matrix to become exposed, in turn activating both circulating platelets and the coagulation cascade. ${ }^{14,15}$ Platelet agonists released by circulating platelets recruit and activate further platelets, while tissue factor is locally released and catalyses factor $\mathrm{X}$ conversion into factor Xa, leading to thrombin formation. Thrombin facilitates fibrin formation, crosslinking platelets and stabilizing the thrombus, suggesting a relationship between the two processes. ${ }^{15}$ Clots forming at the vascular wall have similar levels of contribution from both fibrin and platelets, and thrombin appears to play a pivotal role in myocardial necrosis, vascular inflammation and endothelial dysfunction. ${ }^{16}$ Antiplatelet therapy inhibits the aggregation of platelets activated through contact with collagen in the exposed vessel wall. Anticoagulation reduces the risk of clot formation by suppressing the direct activation of platelets and the generation of fibrin via the coagulation cascade. ${ }^{14,15}$

Factor Xa has a dual-pathway function by reducing thrombin generation and potentially showing complementary favourable activity, such as anti-inflammatory effects and endothelial protection (-Fig. 1). There is recognized therapeutic potential in targeting this dual pathway to reduce the risk of atherothrombotic events, through combining antiplatelet therapy with anticoagulation. Preclinical data have shown that the combination of rivaroxaban (at a dose equivalent to the trough plasma concentration after rivaroxaban $2.5 \mathrm{mg}$ bid in humans) with single- or dualantiplatelet therapy (DAPT) resulted in improved antithrombotic activity compared with rivaroxaban or antiplatelet therapy alone. ${ }^{17}$ The effects of rivaroxaban were strongest on the dynamics of thrombin generation. The combination of rivaroxaban and ticagrelor further reduced thrombin $\mathrm{C}_{\max }$ and mean velocity, and prolonged thrombin $\mathrm{T}_{\max }$, compared with either agent alone. The combination of rivaroxaban, ticagrelor and acetylsalicylic acid (ASA) showed another slight improvement in the effects compared with the rivaroxaban and ticagrelor combination. Similar synergistic effects were also seen with rivaroxaban and ticagrelor for the inhibition of tissue factor-induced platelet aggregation. ${ }^{17}$

Atherothrombosis is pathophysiologically characterized by a diseased endothelium with a low grade of inflammation, which needs continuous and lasting protection. ${ }^{18,19}$ Reduction of long-term CV risk is achieved by a comprehensive

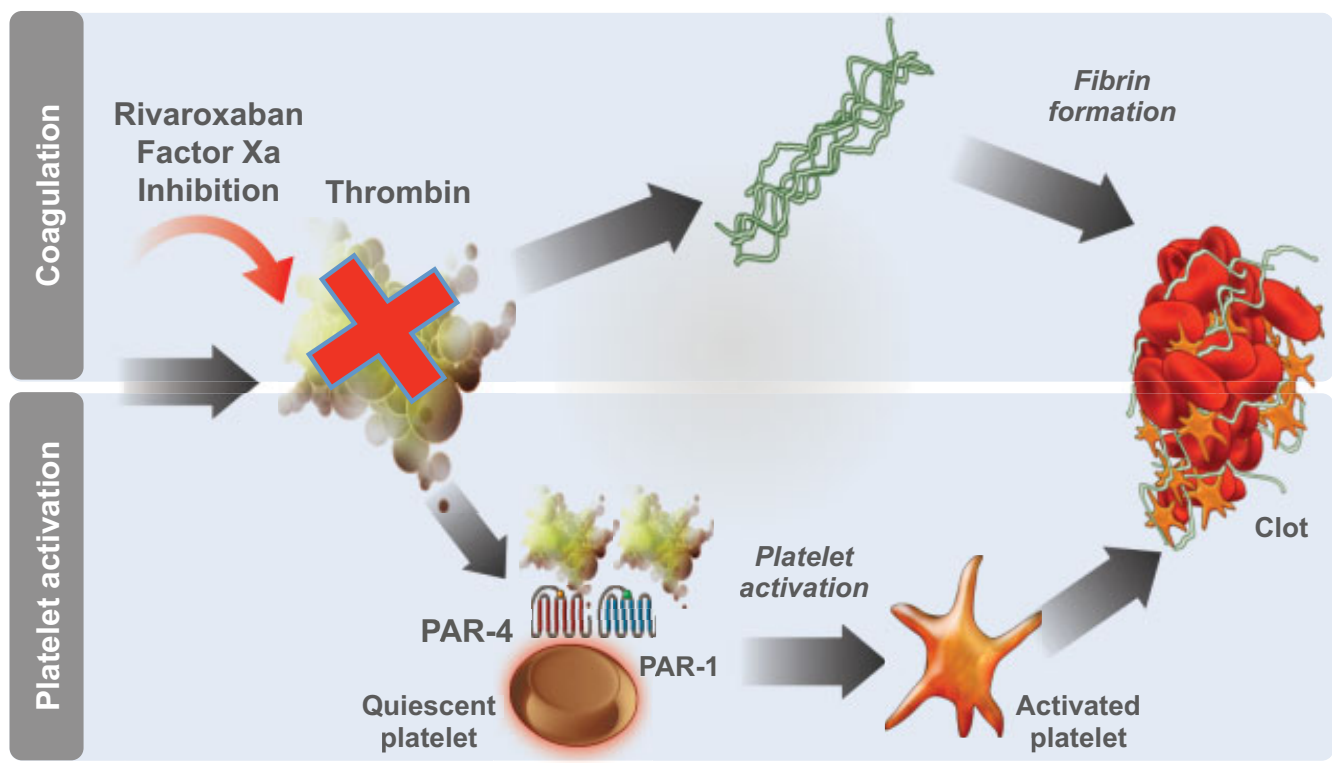

Fig. 1 Rivaroxaban targets essential components of atherothrombosis to provide vascular protection. Adapted with permission (Copyright $\odot$ 2009 European Society of Cardiology. All rights reserved). ${ }^{71}$ PAR, protease-activated receptor. 
strategy, including the use of antithrombotics and lipid- or glucose-lowering drugs. ${ }^{20}$ However, protection conferred with antiplatelets is likely to be limited and individual patient variability exists in response to these drugs. ${ }^{21}$ On the other hand, rivaroxaban $(2.5 \mathrm{mg}$ twice-daily [bid] and $5 \mathrm{mg}$ bid) has been shown to significantly reduce mortality and morbidity in patients with ACS, including those presenting with heart failure (HF). ${ }^{22,23}$

Targeted inhibition of thrombin generation with rivaroxaban in patients with CAD (with or without ischaemic HF) or PAD may stabilize multiple pathophysiological processes, i.e. reduce pro-inflammatory cytokines, reduce restenosis/narrowing of blood vessels and prevent atherosclerotic plaque progression/destabilization (via inhibition of protease-activated receptor [PAR]- $1 / 2$ by factor Xa or inhibition of PAR-1/4 by factor II), ${ }^{24}$ thereby reducing mortality and morbidity. The development program of rivaroxaban in vascular protection aims to further evaluate the role of this factor Xa inhibitor in the prevention of atherothrombotic events ( - Table 1 ). This article reviews the needs of specific groups of patients who have been included in three key randomized controlled trials (RCTs) involving rivaroxaban:

- Cardiovascular OutcoMes for People using Anticoagulation StrategieS (COMPASS) trial of patients with CAD and/ or PAD.

- Vascular Outcomes studY of ASA along with rivaroxaban in Endovascular or surgical limb Revascularization for PAD (VOYAGER PAD) trial of patients undergoing peripheral revascularization procedures.

- Cardiovascular Outcome Modification, Measurement AND Evaluation of Rivaroxaban in patients with Heart Failure (COMMANDER HF) trial of patients with HF and CAD.

\section{Need for Vascular Protection in Patients with CAD}

ACS, a key manifestation of CAD, describes a spectrum of clinical symptoms compatible with acute myocardial ischaemia and includes unstable angina, ST-segment elevation MI (STEMI) and non-STEMI (NSTEMI). These manifestations of coronary artery atherosclerosis have a high clinical risk for the patient. ${ }^{25,26}$

Table 1 An overview of the rivaroxaban vascular protection program $^{23,50,51,67,70}$

\begin{tabular}{|l|l|}
\hline Patient population & Trial \\
\hline Acute or unstable CAD & $\begin{array}{l}\text { ATLAS ACS 2-TIMI 51 } \\
\text { (complete) } \\
\text { GEMINI-ACS-1 (complete) }\end{array}$ \\
\hline Acute or unstable PAD & VOYAGER PAD (ongoing) \\
\hline Acute heart failure and CAD & COMMANDER HF (ongoing) \\
\hline Chronic or stable CAD/PAD & COMPASS (complete ${ }^{\text {a }}$ ) \\
\hline
\end{tabular}

Abbreviations: CAD, coronary artery disease; PAD: peripheral arterial disease.

a Pantoprazole arm still ongoing.
Current guidelines from the European Society of Cardiology (ESC) on the management of stable CAD recommend ASA as the cornerstone of pharmacological prevention of arterial thrombosis, with a dosage of 75 to $150 \mathrm{mg} /$ day offering the best risk-benefit ratio. ${ }^{27} \mathrm{ESC} /$ European Association for Cardio-Thoracic Surgery (EACTS) guidelines recommend that in patients with ACS, DAPT with a P2Y $\mathrm{Y}_{12}$ inhibitor combined with ASA is recommended regardless of initial treatment strategy.$^{28}$ Specifically, for ACS in STEMI patients undergoing percutaneous coronary intervention (PCI), ESC/EACTS guidelines recommend DAPT for 1 to 6 month(s) depending on the bleeding risk; for patients in whom the ischaemic risk prevails over the risk of bleeding, a longer DAPT duration may be considered. ${ }^{28}$ Meanwhile, for ACS in NSTEMI patients, DAPT for 12 months is recommended unless contraindicated (e.g. in those with excessive bleeding risk). ${ }^{29}$ Triple therapy, combining ASA with a $\mathrm{P}_{2} \mathrm{Y}_{12}$ inhibitor and an oral anticoagulant (OAC), is recommended in STEMI patients if there is a clear need for anticoagulation (e.g. atrial fibrillation $[\mathrm{AF}]$ and $\mathrm{CHA}_{2} \mathrm{DS}_{2}$-VASc score $\geq 2$ or mechanical valve prosthesis); the duration of DAPT should be as short as possible to minimize bleeding risk. ${ }^{30}$

A large meta-analysis of more than 25,000 ACS patients previously underlined the increased bleeding risk resulting from ASA being combined with warfarin versus ASA alone, although there was a significant clinical benefit in terms of reducing major adverse events. ${ }^{31}$ In the Clopidogrel for High Atherothrombotic Risk and Ischaemic Stabilization, Management, and Avoidance (CHARISMA) trial of patients with clinically evident $\mathrm{CV}$ disease or who were at high risk of atherothrombotic events, addition of clopidogrel to ASA did not reduce the risk of stroke, MI or CV death. ${ }^{32}$ Recent trials have studied the efficacy of new antiplatelet agents. The Thrombin Receptor Antagonist for Clinical Event Reduction in Acute Coronary Syndrome (TRACER) trial showed that compared with placebo, vorapaxar did not significantly reduce the primary efficacy endpoint of $\mathrm{CV}$ death, MI, stroke, recurrent ischaemia with hospitalization or urgent coronary revascularization when used in combination with standard therapy in stable patients with a history of MI, and was also associated with a significant increase in major bleeding and intracranial haemorrhage (ICH). ${ }^{11}$ While long-term therapy with ticagrelor plus ASA reduced the risk of $\mathrm{CV}$ death, MI or stroke in the Prevention of Cardiovascular Events in Patients with Prior Heart Attack Using Ticagrelor Compared with Placebo on a Background of Aspirin-Thrombolysis in Myocardial Infarction 54 (PEGASUS-TIMI 54) trial of patients with prior MI, this was accompanied by an increased risk of major bleeding. ${ }^{33}$

\section{Need for Vascular Protection in Patients with PAD}

Recent ESC/European Society for Vascular Surgery (ESVS) guidelines on the diagnosis and treatment of PAD use this collective term to describe all vascular sites, including the vertebral, carotid, upper extremity, renal, mesenteric and lower extremity vessels, with the term lower extremity 
arterial disease (LEAD) being used for peripheral artery disease specifically. ${ }^{34}$ The American College of Cardiology (ACC) and AHA Practice Guidelines define four categories of PAD: asymptomatic, claudication, critical limb ischaemia (CLI) and acute limb ischaemia (ALI). ${ }^{35-37}$ Patients who present later than 2 weeks following the onset of ALI have CLI. ${ }^{38}$ When considering the spectrum of PAD, ALI is an acute thrombotically mediated event that may be modified by antithrombotic therapy, while CLI describes the long-term sequelae of progressive atherosclerosis in the limbs, which is less likely to be impacted by antithrombotic therapy. ${ }^{39,40}$

The 2012 American College of Chest Physicians (ACCP) guidelines for patients aged $\geq 50$ years with asymptomatic PAD include ASA 75 to $100 \mathrm{mg} /$ day over no therapy for primary prevention of thrombosis in PAD. ${ }^{41}$ For secondary prevention in patients with symptomatic PAD, including before and after peripheral arterial bypass surgery or percutaneous transluminal angioplasty, long-term ASA 75 to 100 $\mathrm{mg} /$ day, or clopidogrel $75 \mathrm{mg} /$ day, is endorsed. ${ }^{41}$ These recommendations are supported in the 2016 ACC/AHA Task Force clinical practice guidelines and the $2017 \mathrm{ESC} /$ ESVS guidelines. ${ }^{34,35}$ The 2017 ESC/ESVS guidelines also include recommendations of DAPT with ASA and clopidogrel for the management of LEAD after infra-inguinal stent implantation and in below-the-knee bypass with a prosthetic graft. ${ }^{34}$ According to the ESC/ESVS guidelines, OAC alone should be considered in patients with PAD who have an indication for OAC (e.g. AF or mechanical prosthetic valve). $\mathrm{OAC}$ is recommended when the $\mathrm{CHA}_{2} \mathrm{DS}_{2}$-VASc score is $\geq 2$ and OAC should be considered in all other patients with PAD and AF. After endovascular revascularization, ASA or clopidogrel should be considered in addition to OAC for at least 1 month if the bleeding risk is low compared with the risk of stent/graft occlusion; if the bleeding risk is high compared with the risk of stent/graft occlusion, OACs alone should be considered. $^{34}$

Even with currently available clinical management guidelines, the residual risk of atherothrombotic events in PAD remains high. Earlier trials suggest a role for low-dose ASA in PAD outpatients with stage I-II PAD, ${ }^{42}$ while more recent findings failed to support ASA use in a variety of PAD populations, such as those with diabetes mellitus and asymptomatic PAD, ${ }^{43}$ those with asymptomatic atherosclerosis $^{44}$ and 5,269 PAD patients identified in a metaanalysis of 18 trials. $^{45}$

In a subgroup analysis of PAD patients $(n=3,096)$ in the CHARISMA trial, clopidogrel added to ASA reduced the risk of MI or hospitalization but did not reduce the risk of stroke, MI or CV death versus placebo + ASA; severe bleeding remained similar between groups. ${ }^{12}$ The PAR-1 antagonist vorapaxar was shown to reduce the need for peripheral revascularization in patients with PAD in the Thrombin Receptor Antagonist in Secondary Prevention of Atherothrombotic Ischaemic Events-Thrombolysis in Myocardial Infarction 50 (TRA 2 P-TIMI 50) trial. ${ }^{46}$ Vorapaxar did not reduce the risk of $C V$ death, MI or stroke but did reduce ALI and peripheral artery revascularization; however, this was accompanied by an increased incidence of bleeding, including significantly higher moderate-to-severe bleeding events. $^{46}$

Robust evidence is still needed to inform PAD management. Adequate trials are lacking and the management of PAD is sometimes extrapolated from CAD data. Previously published clinical studies support a dual role for rivaroxaban in the activation of platelets and the coagulation pathway, indicating that rivaroxaban could have a synergistic effect when used in combination with antiplatelet agents, leading to the potentiation of antithrombotic efficacy. Following the Phase II dose-finding study Anti-Xa Therapy to Lower cardiovascular events in Addition to Standard therapy in Subjects with Acute Coronary Syndrome-Thrombolysis In Myocardial Infarction 46 (ATLAS ACS-TIMI 46), the clinical benefits of rivaroxaban at doses of 2.5 and $5 \mathrm{mg}$ bid were subsequently shown in the ATLAS ACS 2-TIMI 51 trial. Both doses significantly reduced the composite endpoint of death from CV causes, MI or stroke, when used on background therapy (ASA/clopidogrel) in patients with ACS. ${ }^{23,47}$ In subsequent subanalyses, the rivaroxaban $2.5 \mathrm{mg}$ bid dose was associated with reduced stent thrombosis and patient mortality in patients with ACS who received coronary artery stenting, ${ }^{48}$ along with a reduction in CV deaths in patients with a recent STEMI, 22 while both rivaroxaban 2.5 and $5 \mathrm{mg}$ bid reduced MIs, the majority of which were spontaneous. ${ }^{49}$ The COMPASS and VOYAGER PAD studies aim to further elucidate the efficacy of rivaroxaban 2.5 and/or $5 \mathrm{mg}$ bid: COMPASS, terminated early because of overwhelming efficacy, included patients with stable PAD (also including asymptomatic carotid artery stenosis of mild-to-moderate severity), while the ongoing study VOYAGER PAD includes those with highly symptomatic LEAD recently undergoing peripheral revascularization procedures, thus with critical or acute PAD. ${ }^{50,51}$

\section{COMPASS: Rivaroxaban for the Prevention of Major CV Events in CAD or PAD}

COMPASS is a Phase III trial, designed to determine the efficacy and safety of rivaroxaban, rivaroxaban plus ASA or ASA alone, for reducing the risk of MI, stroke and CV death in patients with CAD and/or PAD. ${ }^{52,53}$

COMPASS included 27,395 patients with CAD or PAD receiving a 1:1:1 ratio of rivaroxaban $2.5 \mathrm{mg}$ bid + ASA $100 \mathrm{mg}$ od; rivaroxaban $5 \mathrm{mg}$ bid or ASA $100 \mathrm{mg}$ od. ${ }^{51}$ Another randomized comparison is still ongoing and is comparing pantoprazole with placebo in those patients not receiving a proton-pump inhibitor (the main outcome is upper gastrointestinal complications). The primary efficacy endpoint in the COMPASS primary analysis was the composite of MI, stroke or CV death. Secondary efficacy endpoints were the composite of ischaemic stroke, MI, ALI or death from coronary heart disease; the composite of ischaemic stroke, MI, ALI or CV death; and death from any cause. The primary safety outcome was based on a modification of the International Society on Thrombosis and Haemostasis (ISTH) criteria and included fatal bleeding, symptomatic bleeding in a critical organ or bleeding into a surgical site requiring reoperation, and bleeding leading to hospitalization (includes presentation to an acute care 
facility without overnight stay). All bleeding that led to presentation at an acute care facility or hospitalization was regarded as major. The net clinical benefit outcome was the composite of CV death, stroke, MI, fatal bleeding or symptomatic bleeding into a critical organ. ${ }^{51}$

Patients included were those who met the criteria for CAD or PAD. Those with CAD needed to meet the following criteria: age $\geq 65$ years, or age $<65$ years with documented atherosclerosis or revascularization in $\geq 2$ vascular beds, or $\geq 2$ additional risk factors. Key exclusion criteria included (but were not limited to) a high risk of bleeding; the requirement for DAPT, other non-ASA antiplatelet therapy or OAC therapy; stroke within 1 month or any history of haemorrhagic or lacunar stroke; severe HF with known left ventricular ejection fraction (LVEF) $<30 \%$ or New York Heart Association class III or IV symptoms and an estimated glomerular filtration rate $<15 \mathrm{~mL} / \mathrm{min}^{.}{ }^{51}$

Recruitment for the COMPASS trial began in early 2013 , with an original completion date expected in 2018. However, the rivaroxaban and ASA arm ceased in February 2017, a year ahead of time, on the recommendation of the Data Monitoring Committee, after reaching their pre-specified criteria for superiority. ${ }^{51}$

A total of 602 centres across 33 countries were included in the trial. A total of 9,152, 9,117 and 9,126 patients were included in the rivaroxaban $2.5 \mathrm{mg}$ bid + ASA arm, rivaroxaban $5 \mathrm{mg}$ bid alone arm and ASA alone arm, respectively. The mean age of participants was 68.2 years, and $22 \%$ were women. Lipid-lowering agents were used by $89.8 \%$, and angiotensin-converting enzyme inhibitors or angiotensinreceptor blockers by $71.2 \%$. A total of $90.6 \%$ of the participants had a history of CAD, and $27.3 \%$ had a history of PAD. ${ }^{51}$
The study met its primary endpoint, as the primary outcome was significantly reduced: $4.1 \%$ of patients in the rivaroxaban + ASA group versus 5.4\% in those who received ASA alone (hazard ratio [HR]: 0.76; 95\% confidence interval [CI]: 0.66-0.86; $p<0.001 ;$ - Fig. 2). The incidence of major bleeding was higher in the rivaroxaban + ASA group (3.1\%) compared with $1.9 \%$ for ASA alone (HR: 1.70 ; $95 \%$ CI: $1.40-$ $2.05 ; p<0.001)$; importantly, however, there was no significant difference in intracranial, fatal or critical organ bleeding between these two groups. Secondary composite outcomes (composite of ischaemic stroke, MI, ALI or death from coronary heart disease; and the composite of ischaemic stroke, MI, ALI or CV death) were also significantly more favourable in the rivaroxaban + ASA group versus ASA alone $(p<0.001$ for both). The number of overall deaths was reduced in the rivaroxaban + ASA arm versus the ASA alone $\operatorname{arm}(313$ [3.4\%] vs. 378 [4.1\%]; HR: 0.82; 95\% CI: 0.71-0.96; $p=0.01$ ). While significant benefits were observed following the use of rivaroxaban $2.5 \mathrm{mg}$ bid + ASA, the use of rivaroxaban $5 \mathrm{mg}$ bid alone did not lead to a significant reduction in the primary outcome compared with ASA alone ( 4.9 vs. $5.4 \%$; HR: $0.90 ; 95 \%$ CI: $0.79-1.03 ; p=0.12$ ), while major bleeding was significantly higher compared with ASA (2.8 vs. $1.9 \%$; HR: $1.51 ; 95 \% \mathrm{CI}: 1.25-1.84 ; p<0.001$ ). The risk of the composite net clinical benefit outcome of $\mathrm{CV}$ death, stroke, MI, fatal bleeding or symptomatic bleeding into a critical organ was lower with rivaroxaban + ASA than with ASA alone (431 patients [4.7\%] vs. 534 patients [5.9\%]; HR: $0.80 ; 95 \%$ CI: $0.70-0.91 ; p<0.001$ ); however, this was not the case for rivaroxaban alone versus ASA alone. ${ }^{51}$

Rivaroxaban + ASA versus ASA alone also showed consistent benefits across subgroups studied, including those

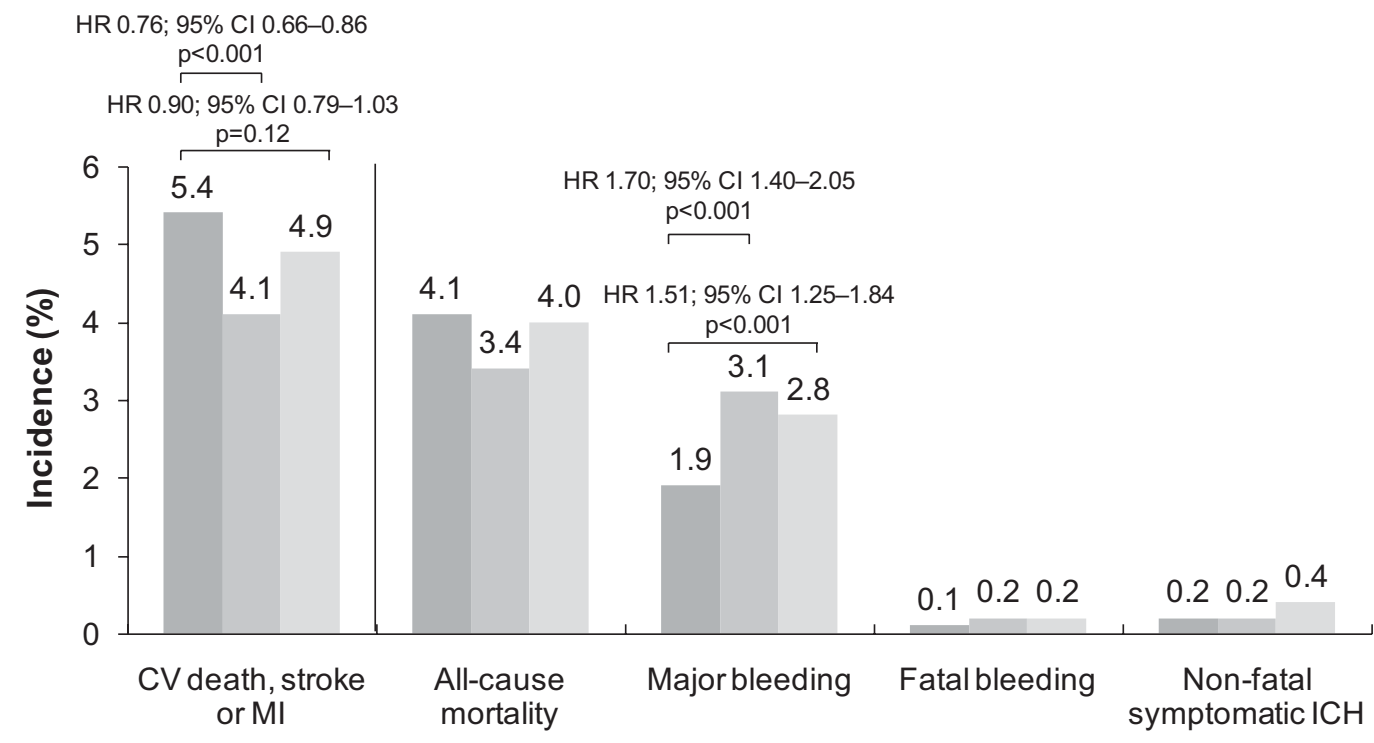

\section{Composite endpoint}

\section{ASA $100 \mathrm{mg}$ od \\ Rivaroxaban $2.5 \mathrm{mg}$ bid + ASA \\ Rivaroxaban $5 \mathrm{mg}$ bid}

Fig. 2 Clinical outcomes from COMPASS primary analysis. ${ }^{51}$ ASA, acetylsalicylic acid; bid, twice daily; Cl, confidence interval; CV, cardiovascular; HR, hazard ratio; ICH, intracranial bleeding; MI, myocardial infarction; od, once daily. 
with either CAD or PAD. Of the PAD patients included in COMPASS $(n=7,470)$, similar outcomes were observed with regards to the clinical efficacy of rivaroxaban, where rivaroxaban $2.5 \mathrm{mg}$ bid + ASA was significantly superior to ASA alone in reducing major adverse $\mathrm{CV}$ events (MACE) or major adverse limb events (MALE) or major amputation (HR: 0.69; 95\% CI: $0.56-0.85 ; p=0.0003) .{ }^{54}$ While there was an increase in major bleeding with rivaroxaban + ASA, there was no significant increase in fatal or critical organ bleeding. MACE or MALE or major amputation outcomes were also consistent across PAD subgroups (symptomatic PAD, PAD lower extremities and carotid artery disease) where rivaroxaban $2.5 \mathrm{mg}$ bid + ASA was favoured over ASA alone. ${ }^{54}$ There were several limitations of COMPASS. Patients with a recent stroke or previous haemorrhagic or lacunar stroke were excluded; however, of those enrolled, 1,032 had a history of stroke, and the benefits of the rivaroxaban and ASA combination in preventing cardiovascular death, stroke or MI were consistent in these patients. Furthermore, the rivaroxaban and ASA combination resulted in a lower rate of ischaemic stroke than ASA alone. The investigators did not specifically record statin use or low-density lipoprotein cholesterol levels at baseline, and the trial protocol did not specifically emphasize aggressive use of secondary prevention therapies to lower blood pressure and cholesterol levels. However, the results supported the conclusion that the benefits of combination therapy were additive to those of other proven secondary preventive therapies. As the trial was stopped early, it could be suggested that the efficacy observed may overestimate the treatment effect. However, before the time of stopping, the data and safety monitoring board had observed a progressive increase in benefit of the rivaroxaban and ASA combination for more than 1 year. ${ }^{51}$

Data from COMPASS present substantial benefits with rivaroxaban $2.5 \mathrm{mg}$ bid in combination with ASA in terms of improved survival and reductions in stroke and $\mathrm{MI}$ in patients with stable CAD or PAD. These landmark findings support the concept of combined antithrombotic therapy with an anticoagulant and an antiplatelet agent to ultimately provide long-lasting vascular protection.

\section{VOYAGER PAD: Rivaroxaban in Patients with PAD Undergoing Peripheral Revascularization Procedures}

VOYAGER PAD is a Phase III, randomized, double-blind, placebo-controlled trial, designed to determine the safety and efficacy of a dual-pathway approach versus standard of care (ASA alone) for the reduction of thrombotic vascular events in subjects after peripheral revascularization procedures ( - Fig. 3). ${ }^{50}$ The trial includes three strata of patients, all with infrainguinal procedures: angioplasty (with/or without stent) receiving clopidogrel on top of ASA (DAPT); angioplasty (with/or without stent) receiving no clopidogrel; and surgery, including bypass surgery or thromboendarterectomy. Approximately 6,500 patients will be randomized in a ratio of $1: 1$ to receive rivaroxaban $2.5 \mathrm{mg}$ bid plus ASA $100 \mathrm{mg}$ od, or ASA $100 \mathrm{mg}$ od alone. ${ }^{50}$ The primary efficacy outcome is the composite of MI, ischaemic stroke, CV death, ALI and major amputation due to PAD. The primary safety endpoint is major bleeding, as assessed by the Thrombolysis in Myocardial Infarction score. ${ }^{50}$

\section{VOYAGER PAD}
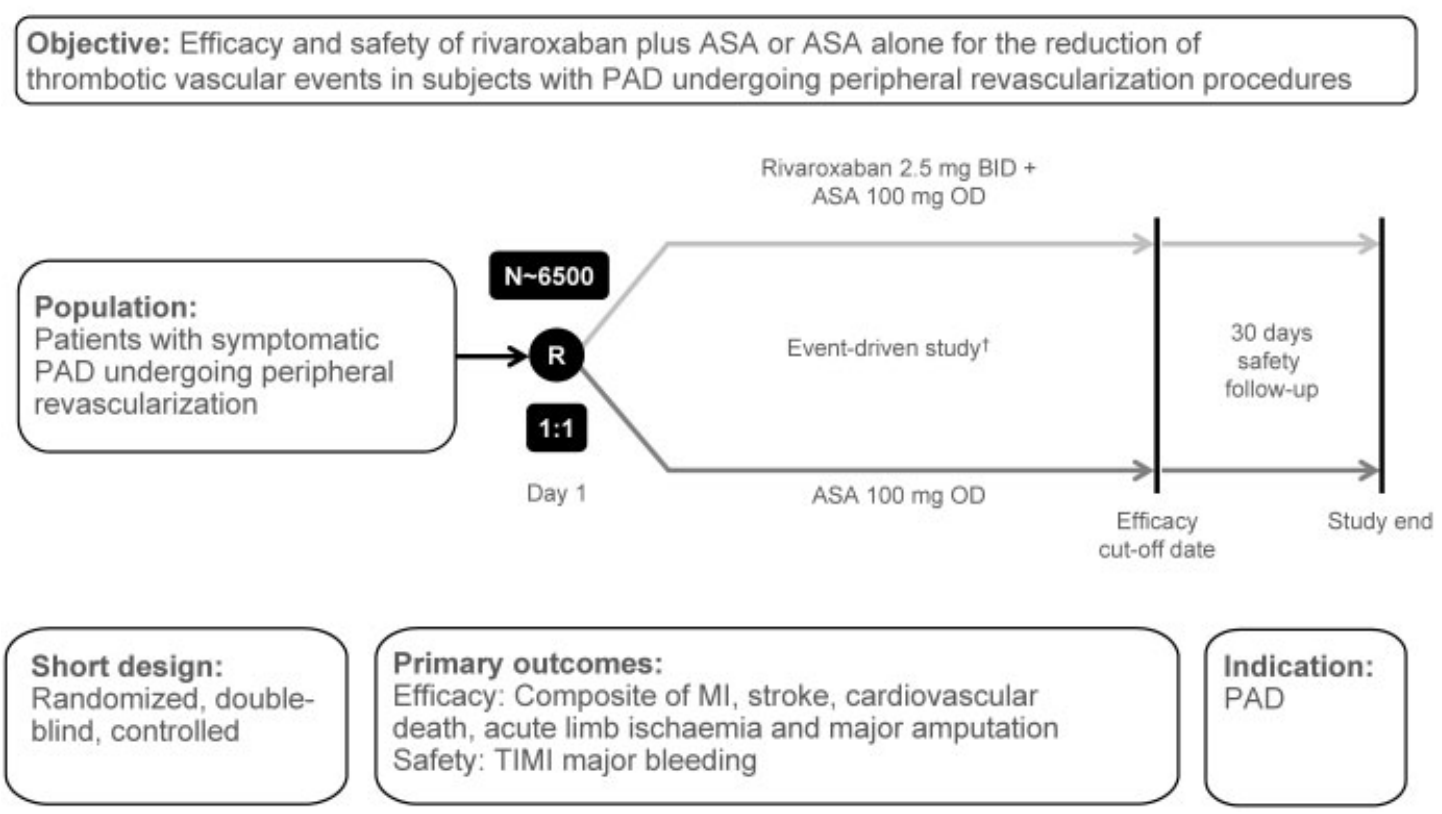

Fig. 3 VOYAGER PAD trial design. ${ }^{50}$ 'Mean treatment duration approximately 30 months. ASA, acetylsalicylic acid; bid, twice-daily; od, once daily; PAD, peripheral arterial disease; MI, myocardial infarction; R, randomized; TIMI, thrombolysis in myocardial infarction. 
Key inclusion criteria for VOYAGER PAD are documented moderate-or-severe symptomatic lower limb PAD and recent successful peripheral infra-inguinal revascularization (within the last 10 days prior to revascularization), and age $\geq 50$ years. Exclusion criteria include revascularization for asymptomatic PAD or mild claudication; revascularization to treat an asymptomatic restenosis of a bypass graft; prior revascularization on the index leg within 10 days of the qualifying revascularization planned use of additional antiplatelet agents other than clopidogrel and ASA following the revascularization procedure and planned dual-antiplatelet treatment beyond 6 months following the revascularization procedure. ${ }^{50}$ VOYAGER PAD began enrolling in late 2015, with results expected in 2019.

\section{Need for Vascular Protection in Patients with CAD and HF}

The underlying cause of HF has changed from hypertension to CAD in recent decades. ${ }^{55,56}$ Approximately $65 \%$ of patients admitted to hospital for HF have CAD as the underlying cause. CAD has also been shown to be independently associated with worsening long-term prognosis for patients with $\mathrm{HF}^{56}$ Coronary artery atherothrombosis, resulting in myocardial ischaemia, is a common cause of $\mathrm{HF}$ with a reduced ejection fraction (HFrEF, also known as systolic HF), where the heart muscle is not able to contract adequately. The pathogenesis of increased thrombosis in patients with HF may be due to reduced blood flow as a result of low cardiac output, decreased physical activity and a hypercoagulable state, as shown by an increase in platelet activation, plasma viscosity, fibrinogen, fibrin, D-dimer and von Willebrand factor. ${ }^{57-59}$ Thrombin appears to play a pivotal role in fibrinolysis, inflammation and endothelial dysfunction in this setting ${ }^{57}$ and, therefore, anticoagulants may be important in the prevention of $\mathrm{CV}$ events in $\mathrm{HF}$ for patients in sinus rhythm.

Guidance supporting the use of antiplatelets or anticoagulants other than ASA in the prevention of CV events in patients with HF is scarce. The 2013 American College of Cardiology Foundation (ACCF) and the AHA Task Force guidelines on the management of patients with HF recommend non-vitamin $\mathrm{K}$ antagonist oral anticoagulants (NOACs) in patients with $\mathrm{HF}$ and $\mathrm{AF}$, and an additional risk factor for stroke. ${ }^{26}$ NOACs are not recommended in patients with HFrEF without AF, a prior thromboembolic event or a known cardioembolic source. ${ }^{26}$ The 2012 ESC guidelines do not recommend anticoagulation for patients with HFrEF, unless there is concomitant $\mathrm{AF}^{60}{ }^{60}$ Although anticoagulants may have a role in preventing $\mathrm{CV}$ events in patients with $\mathrm{HF}$ in sinus rhythm, at present RCTs in this clinical setting have been inconclusive. ${ }^{61}$

As thrombotic events are more common in patients with HF, several studies have investigated oral anticoagulation treatment strategies. Analyses from the Warfarin/Aspirin Study in Heart failure (WASH) trial found that warfarin antithrombotic therapy does not modify mortality or vascular events in patients with HF in sinus rhythm, ${ }^{62}$ while findings from the Heart failure Long-term Antithrombotic Study (HELAS) had inconclusive findings owing to the small number of patients recruited. ${ }^{63}$ These results were confirmed in a Cochrane review, which concluded that although OACs are indicated in certain groups of patients with HF, the available data do not support their routine use in patients with $\mathrm{HF}$ in sinus rhythm. ${ }^{64}$ In another recent study of patients with HF in sinus rhythm, the Warfarin versus Aspirin Reduced Cardiac Ejection Fraction (WARCEF) trial showed no significant difference between warfarin and ASA in the primary composite outcome of ischaemic stroke, intracerebral haemorrhage or death from any cause. The reduced risk of ischaemic stroke observed with warfarin was accompanied by an increased risk in major bleeding. ${ }^{61}$

Subgroup analyses in ATLAS ACS 2-TIMI 51 showed consistent benefits of rivaroxaban $2.5 \mathrm{mg}$ bid in those with congestive HF. ${ }^{65}$ Rivaroxaban $2.5 \mathrm{mg}$ bid is approved in Europe for the treatment of biomarker-positive patients after an $\mathrm{ACS}^{66}$ and was chosen as the dose to investigate in the COMMANDER HF trial as the most likely to offer an optimal combination of safety and efficacy, by inclusion in a targeted antithrombotic strategy in patients with a recent exacerbation of HFrEF and concomitant CAD.

\section{COMMANDER HF: Rivaroxaban in Patients with HF and Significant CAD following an Exacerbation of $\mathbf{H F}$}

COMMANDER HF is an international, Phase III, prospective, randomized, double-blind, placebo-controlled, event-driven, parallel-group comparison of the efficacy and safety of rivaroxaban with placebo and standard of care, to reduce the risk of MI, stroke or death in patients with documented symptomatic HF and evidence of significant CAD. ${ }^{67}$ Approximately 5,000 patients will be randomized to receive rivaroxaban $2.5 \mathrm{mg}$ bid plus standard of care versus placebo plus standard of care ( - Fig. 4). Patients will take the study drug until the targeted number of events has been predicted to have occurred and will be followed up for 7 to 31 months. ${ }^{67}$

The inclusion criteria include a recent symptomatic exacerbation of HF, increased plasma concentrations of natriuretic peptides ( $\mathrm{N}$-terminal pro-B-type natriuretic peptide $\geq 800 \mathrm{pg} / \mathrm{mL}$ or B-type natriuretic peptide $\geq 200 \mathrm{pg} / \mathrm{mL}$ ), with an LVEF $\leq 40 \%$ and significant CAD. The exclusion criteria include requirement of anticoagulation for AF or for other clinical conditions. ${ }^{67}$

Following an index event, such as a hospital admission, unscheduled outpatient treatment or worsening HF, patients will be randomized in a ratio of 1:1 to rivaroxaban or placebo (with standard of care). The primary efficacy outcome event is a composite of all-cause mortality, MI or stroke. The principal safety outcome event is the composite of fatal bleeding or bleeding into a critical space that has the potential for permanent disability, bleeding events requiring hospitalization and major bleeding events, according to ISTH criteria. ${ }^{67}$

COMMANDER HF began enrolling patients in Q3 2013, with results expected in 2018. It is the first prospective study of an NOAC in HF and will provide important information on the use of rivaroxaban following an HF event in an HFrEF patient population with CAD. ${ }^{67,68}$ 


\section{COMMANDER HF}

Objective: Efficacy and safety of rivaroxaban or ASA, on a background of single of dual antiplatelet therapy, for reducing the risk of MI, stroke or death in HF with CAD

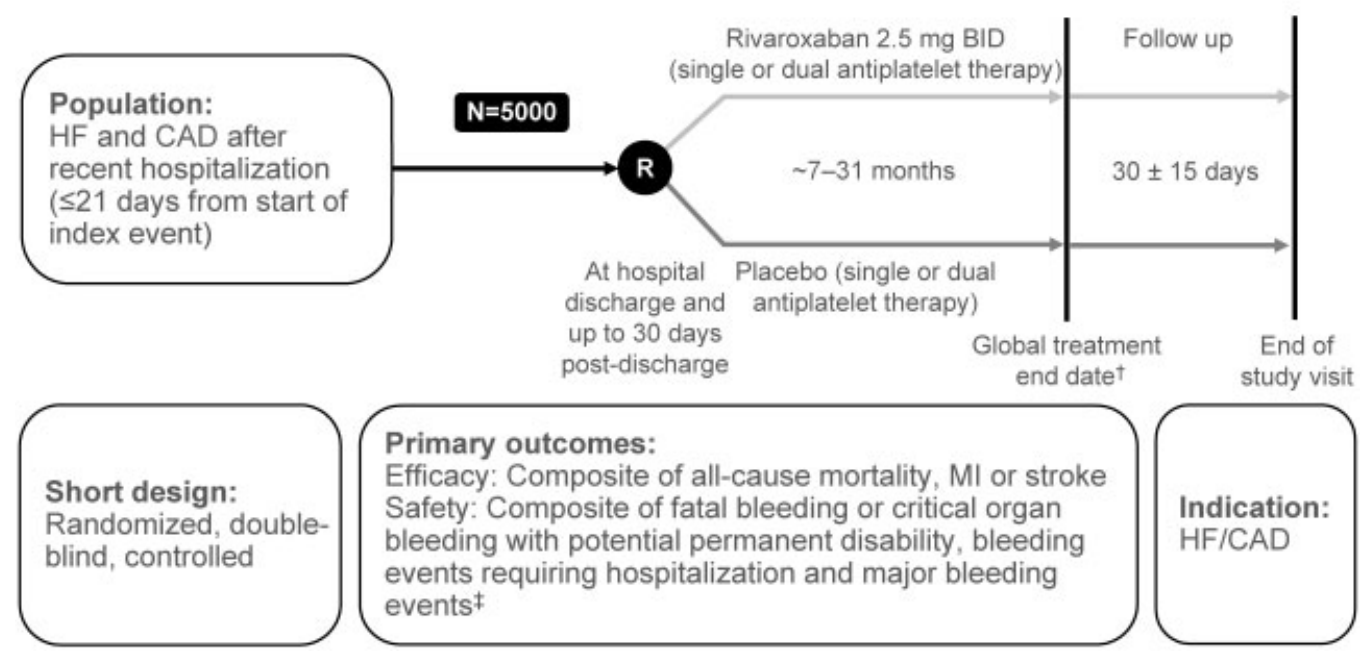

Fig. 4 COMMANDER HF trial design. ${ }^{67,68}{ }^{\dagger}$ The date when 984 primary efficacy outcome events are predicted to have occurred. ${ }^{\ddagger}$ According to the International Society on Thrombosis and Haemostasis (ISTH) bleeding criteria. ASA, acetylsalicylic acid; bid, twice daily; CAD, coronary artery disease; HF, heart failure; MI, myocardial infarction; R, randomized.

\section{Conclusion}

Despite advances in medicine, the burden of $\mathrm{CV}$ disease continues to increase because of the aging population. A significant proportion of patients with CV disease have polyvascular disease or HF, which exacerbates their risk even further. Despite intensive antiplatelet therapy, which has been traditionally accepted as the standard of care in this setting, patients with CAD and PAD exhibit a high residual risk of atherothrombotic events. Antithrombotic therapy composed of anticoagulants in addition to antiplatelets may present an alternative approach. Importantly, the net clinical benefit of rivaroxaban $2.5 \mathrm{mg}$ bid combined with ASA has been clearly shown in the landmark COMPASS trial. In November 2017, rivaroxaban in combination with ASA was filed for approval by the European Medicines Agency (EMA) for the treatment of patients with CAD or PAD. ${ }^{69}$ While the benefits of this new approach are clear, integration into daily practice may be slowed down by barriers such as bleeding concerns, cost and identification of appropriate patients for treatment. Importantly, substantial changes will need to be implemented into practice to make way for the new treatment amongst many other existing antithrombotic options. Developing tools to individualize treatment decisions will be important in terms of implementation in an effort to balance thrombotic and bleeding risks in routine clinical practice. Other contemporary trials of rivaroxaban are ongoing in patients with HF and those undergoing peripheral revascularization procedures-while these findings are still awaited, we must anticipate that redefining the standard of care for CAD patients with a high need for improved CV protection will be a huge barrier to overcome-yet will also carry significant benefits for many.

\section{What is known about this topic?}

- With the ageing population, the burden of cardiovascular disease continues to increase.

- A significant proportion of patients with cardiovascular disease have polyvascular disease or $\mathrm{HF}$, which further exacerbates their risk.

- Despite intensive antiplatelet therapy, patients with CAD and PAD exhibit a high residual risk of atherothrombotic events.

- Clinical trials support the efficacy of rivaroxaban following ACS events.

\section{What does this paper add?}

- This paper reviews ongoing and completed clinical trials from the development programme of rivaroxaban in vascular protection, which hypothesize that antithrombotic therapy comprising NOACs with or without antiplatelet therapy may present therapeutic potential in further reducing the risk of atherothrombotic events.

- Key findings from the Phase III COMPASS trial are discussed, which support the benefits of rivaroxaban $2.5 \mathrm{mg}$ bid in combination with ASA in terms of improved survival and reductions in stroke and $\mathrm{MI}$ in patients with stable CAD or PAD. 


\section{Conflicts of Interest}

All authors confirm that they have had full access to data and contributed to the drafting of the paper. R.B. has received funding from Bayer, BMS, Boehringer Ingelheim, Daiichi-Sankyo and Pfizer for consulting work and speaker bureaus. R.B. also provides research support, as a principal investigator, for Bayer, BMS, Boehringer, Daiichi-Sankyo, Leo Pharma and Portola Pharmaceuticals. F.Z. reports the following: grants to institution from Roche Diagnostics; membership of steering committees of Bayer, Boston Scientific, Janssen, Novartis, Pfizer, ResMed and Takeda; consultant/scientific advisory board membership of Air Liquide, Amgen, CVRx, Servier, St Jude and Stealth Peptide; speaker fees from Mitsubishi; and has stocks in CardioRenal Diagnostics and CVCT.

\section{Grants or Other Financial Support}

Editorial support funded by Bayer AG.

\section{Acknowledgement}

The authors would like to acknowledge Kelly Farrell at Ketchum (Inspired Science), who provided editorial support with funding from Bayer AG.

\section{References}

1 Odden MC, Coxson PG, Moran A, Lightwood JM, Goldman L, Bibbins-Domingo $\mathrm{K}$. The impact of the aging population on coronary heart disease in the United States. Am J Med 2011; 124(09):827-33.e5

2 World Health Organization. Top 10 causes of death. Fact Sheet No. 310. Available at: http://www.who.int/mediacentre/factsheets/ fs310/en/. Accessed February 21, 2018

3 GBD 2013 Mortality and Causes of Death Collaborators. Global, regional, and national age-sex specific all-cause and cause-specific mortality for 240 causes of death, 1990-2013: a systematic analysis for the Global Burden of Disease Study 2013. Lancet 2015;385(9963):117-171

4 Benjamin EJ, Blaha MJ, Chiuve SE, et al; American Heart Association Statistics Committee and Stroke Statistics Subcommittee. Heart disease and stroke statistics-2017 update: a report from the American Heart Association. Circulation 2017;135(10):e146-e603

5 Fowkes FG, Rudan D, Rudan I, et al. Comparison of global estimates of prevalence and risk factors for peripheral artery disease in 2000 and 2010: a systematic review and analysis. Lancet 2013; 382(9901):1329-1340

6 Serrano Hernando FJ, Martín Conejero A. Peripheral artery disease: pathophysiology, diagnosis and treatment [in Spanish]. Rev Esp Cardiol 2007;60(09):969-982

7 Kullo IJ, Rooke TW. CLINICAL PRACTICE. Peripheral artery disease. N Engl J Med 2016;374(09):861-871

8 Criqui MH, Langer RD, Fronek A, et al. Mortality over a period of 10 years in patients with peripheral arterial disease. $\mathrm{N}$ Engl $\mathrm{J}$ Med 1992;326(06):381-386

9 Bhatt DL, Steg PG, Ohman EM, et al; REACH Registry Investigators. International prevalence, recognition, and treatment of cardiovascular risk factors in outpatients with atherothrombosis. JAMA 2006;295(02):180-189

10 Bhatt DL, Fox KA, Hacke W, et al; CHARISMA Investigators. Clopidogrel and aspirin versus aspirin alone for the prevention of atherothrombotic events. N Engl J Med 2006;354(16):1706-1717

11 Tricoci P, Huang Z, Held C, et al; TRACER Investigators. Thrombinreceptor antagonist vorapaxar in acute coronary syndromes. $\mathrm{N}$ Engl J Med 2012;366(01):20-33
12 Cacoub PP, Bhatt DL, Steg PG, Topol EJ, Creager MA; CHARISMA Investigators. Patients with peripheral arterial disease in the CHARISMA trial. Eur Heart J 2009;30(02):192-201

13 Bonaca MP, Scirica BM, Creager MA, et al. Vorapaxar in patients with peripheral artery disease: results from TRA2 ${ }^{\circ}$ P-TIMI 50. Circulation 2013;127(14):1522-1529

14 Hess CN, Norgren L, Ansel GM, et al. A structured review of antithrombotic therapy in peripheral artery disease with a focus on revascularization: a TASC (InterSociety Consensus for the Management of Peripheral Artery Disease) initiative. Circulation 2017;135(25):2534-2555

15 Stachon P, Ahrens I, Bode C, Zirlik A. Dual pathway therapy in acute coronary syndrome. J Thromb Thrombolysis 2016;42(02): 254-260

16 Ten Cate H, Hemker HC. Thrombin generation and atherothrombosis: what does the evidence indicate? J Am Heart Assoc 2016;5 (08): $\mathrm{e} 003553$

17 Perzborn E, Heitmeier S, Laux V. Effects of rivaroxaban on platelet activation and platelet-coagulation pathway interaction: in vitro and in vivo studies. J Cardiovasc Pharmacol Ther 2015;20(06): 554-562

18 Badimon L, Storey RF, Vilahur G. Update on lipids, inflammation and atherothrombosis. Thromb Haemost 2011;105(Suppl 1): S34-S42

19 Borissoff JI, Spronk HM, ten Cate H. The hemostatic system as a modulator of atherosclerosis. N Engl J Med 2011;364(18): $1746-1760$

20 Cortés-Beringola A, Fitzsimons D, Pelliccia A, Moreno G, MartínAsenjo R, Bueno H. Planning secondary prevention: room for improvement. Eur J Prev Cardiol 2017;24(3 Suppl):22-28

21 Weitz JI, Eikelboom JW, Samama MM. New antithrombotic drugs: Antithrombotic Therapy and Prevention of Thrombosis, 9th ed: American College of Chest Physicians Evidence-Based Clinical Practice Guidelines. Chest 2012;141(2 Suppl):e120S-e151S

22 Mega JL, Braunwald E, Murphy SA, et al. Rivaroxaban in patients stabilized after a ST-segment elevation myocardial infarction: results from the ATLAS ACS-2-TIMI-51 trial (Anti-Xa Therapy to Lower Cardiovascular Events in Addition to Standard Therapy in Subjects with Acute Coronary Syndrome-Thrombolysis In Myocardial Infarction-51). J Am Coll Cardiol 2013;61(18):1853-1859

23 Mega JL, Braunwald E, Wiviott SD, et al; ATLAS ACS 2-TIMI 51 Investigators. Rivaroxaban in patients with a recent acute coronary syndrome. N Engl J Med 2012;366(01):9-19

24 Spronk HM, de Jong AM, Crijns HJ, Schotten U, Van Gelder IC, Ten Cate H. Pleiotropic effects of factor Xa and thrombin: what to expect from novel anticoagulants. Cardiovasc Res 2014;101(03): 344-351

25 Kumar A, Cannon CP. Acute coronary syndromes: diagnosis and management, part I. Mayo Clin Proc 2009;84(10):917-938

26 Yancy CW, Jessup M, Bozkurt B, et al; Writing Committee Members; American College of Cardiology Foundation/American Heart Association Task Force on Practice Guidelines. 2013 ACCF/AHA guideline for the management of heart failure: a report of the American College of Cardiology Foundation/American Heart Association Task Force on practice guidelines. Circulation 2013; 128(16):e240-e327

27 Montalescot G, Sechtem U, Achenbach S, et al; Task Force Members; ESC Committee for Practice Guidelines; Document Reviewers. 2013 ESC guidelines on the management of stable coronary artery disease: the Task Force on the management of stable coronary artery disease of the European Society of Cardiology. Eur Heart J 2013;34(38):2949-3003

28 Valgimigli M, Bueno H, Byrne RA, et al; ESC Scientific Document Group; ESC Committee for Practice Guidelines (CPG); ESC National Cardiac Societies. 2017 ESC focused update on dual antiplatelet therapy in coronary artery disease developed in collaboration with EACTS: The Task Force for dual antiplatelet therapy in coronary artery disease of the European Society of 
Cardiology (ESC) and of the European Association for CardioThoracic Surgery (EACTS). Eur Heart J 2018;39(03):213-260

29 Roffi M, Patrono C, Collet JP, et al; Management of Acute Coronary Syndromes in Patients Presenting without Persistent ST-Segment Elevation of the European Society of Cardiology. 2015 ESC Guidelines for the management of acute coronary syndromes in patients presenting without persistent ST-segment elevation: Task Force for the Management of Acute Coronary Syndromes in Patients Presenting without Persistent ST-Segment Elevation of the European Society of Cardiology (ESC). Eur Heart J 2016;37 (03):267-315

30 Steg PG, James SK, Atar D, et al; Task Force on the Management of ST-Segment Elevation Acute Myocardial Infarction of the European Society of Cardiology (ESC). ESC Guidelines for the management of acute myocardial infarction in patients presenting with ST-segment elevation. Eur Heart J 2012;33(20):2569-2619

31 Andreotti F, Testa L, Biondi-Zoccai GG, Crea F. Aspirin plus warfarin compared to aspirin alone after acute coronary syndromes: an updated and comprehensive meta-analysis of 25,307 patients. Eur Heart J 2006;27(05):519-526

32 Berger JS, Bhatt DL, Steg PG, et al. Bleeding, mortality, and antiplatelet therapy: results from the Clopidogrel for High Atherothrombotic Risk and Ischemic Stabilization, Management, and Avoidance (CHARISMA) trial. Am Heart J 2011;162(01):98-105. e1

33 Bonaca MP, Bhatt DL, Cohen M, et al; PEGASUS-TIMI 54 Steering Committee and Investigators. Long-term use of ticagrelor in patients with prior myocardial infarction. N Engl J Med 2015; 372(19):1791-1800

34 Aboyans V, Ricco JB, Bartelink MEL, et al. ESC Guidelines on the Diagnosis and Treatment of Peripheral Arterial Diseases, in collaboration with the European Society for Vascular Surgery (ESVS): Document covering atherosclerotic disease of extracranial carotid and vertebral, mesenteric, renal, upper and lower extremity arteries. Endorsed by: the European Stroke Organization (ESO), The Task Force for the Diagnosis and Treatment of Peripheral Arterial Diseases of the European Society of Cardiology (ESC) and of the European Society for Vascular Surgery (ESVS). Eur Heart J 2017; doi: 10.1093/eurheartj/ehx095 [Epub ahead of print]

35 Gerhard-Herman MD, Gornik HL, Barrett C, et al. 2016 AHA/ACC Guideline on the Management of Patients With Lower Extremity Peripheral Artery Disease: Executive Summary: A Report of the American College of Cardiology/American Heart Association Task Force on Clinical Practice Guidelines. Circulation 2017;135(12): e686-e725

36 Hirsch AT, Haskal ZJ, Hertzer NR, et al (Writing Committee Members); Antman EM, Smith Jr SC, Adams CD, et al (Task Force Members). ACC/AHA 2005 Practice Guidelines for the management of patients with peripheral arterial disease (lower extremity, renal, mesenteric, and abdominal aortic). Circulation 2006; 113(11):e463-e654

37 Rooke TW, Hirsch AT, Misra S, et al; American College of Cardiology Foundation Task Force; American Heart Association Task Force. Management of patients with peripheral artery disease (compilation of 2005 and 2011 ACCF/AHA Guideline Recommendations): a report of the American College of Cardiology Foundation/American Heart Association Task Force on Practice Guidelines. J Am Coll Cardiol 2013;61(14):1555-1570

$38 \mathrm{Keo} \mathrm{HH}$, Duval S, Baumgartner I, et al. The FReedom from Ischemic Events-New Dimensions for Survival (FRIENDS) registry: design of a prospective cohort study of patients with advanced peripheral artery disease. BMC Cardiovasc Disord 2013;13:120

39 Baril DT, Ghosh K, Rosen AB. Trends in the incidence, treatment, and outcomes of acute lower extremity ischemia in the United States Medicare population. J Vasc Surg 2014;60(03): 669-77.e2

40 Beard JD. ABC of arterial and venous disease: chronic lower limb ischaemia. BMJ 2000;320(7238):854-857
41 Alonso-Coello P, Bellmunt S, McGorrian C, et al. Antithrombotic therapy in peripheral artery disease: Antithrombotic Therapy and Prevention of Thrombosis, 9th ed: American College of Chest Physicians Evidence-Based Clinical Practice Guidelines. Chest 2012;141(2, Suppl):e669S-e690S

42 Catalano M, Born G, Peto R; Critical Leg Ischaemia Prevention Study (CLIPS) Group. Prevention of serious vascular events by aspirin amongst patients with peripheral arterial disease: randomized, double-blind trial. J Intern Med 2007;261(03):276-284

43 Belch J, MacCuish A, Campbell I, et al; Prevention of Progression of Arterial Disease and Diabetes Study Group; Diabetes Registry Group; Royal College of Physicians Edinburgh. The prevention of progression of arterial disease and diabetes (POPADAD) trial: factorial randomised placebo controlled trial of aspirin and antioxidants in patients with diabetes and asymptomatic peripheral arterial disease. BMJ 2008;337:a1840

44 Fowkes FG, Price JF, Stewart MC, et al; Aspirin for Asymptomatic Atherosclerosis Trialists. Aspirin for prevention of cardiovascular events in a general population screened for a low ankle brachial index: a randomized controlled trial. JAMA 2010;303(09):841-848

45 Berger JS, Krantz MJ, Kittelson JM, Hiatt WR. Aspirin for the prevention of cardiovascular events in patients with peripheral artery disease: a meta-analysis of randomized trials. JAMA 2009; 301(18):1909-1919

46 Bonaca MP, Creager MA, Olin J, et al. Peripheral revascularization in patients with peripheral artery disease with vorapaxar: insights from the TRA $2^{\circ}$ P-TIMI 50. JACC Cardiovasc Interv 2016; 9(20):2157-2164

47 Mega JL, Braunwald E, Mohanavelu S, et al; ATLAS ACS-TIMI 46 study group. Rivaroxaban versus placebo in patients with acute coronary syndromes (ATLAS ACS-TIMI 46): a randomised, doubleblind, phase II trial. Lancet 2009;374(9683):29-38

48 Gibson CM, Chakrabarti AK, Mega J, et al; ATLAS-ACS 2 TIMI 51 Investigators. Reduction of stent thrombosis in patients with acute coronary syndromes treated with rivaroxaban in ATLASACS 2 TIMI 51. J Am Coll Cardiol 2013;62(04):286-290

49 Cavender MA, Gibson CM, Braunwald E, et al. The effect of rivaroxaban on myocardial infarction in the ATLAS ACS 2 - TIMI 51 trial. Eur Heart J Acute Cardiovasc Care 2015;4(05):468-474

50 Capell WH, Bonaca MP, Nehler MR, et al. Rationale and design for the Vascular Outcomes study of ASA along with rivaroxaban in endovascular or surgical limb revascularization for peripheral artery disease (VOYAGER PAD). Am Heart J 2018;199:83-91

51 Eikelboom JW, Connolly SJ, Bosch J, et al; COMPASS Investigators. Rivaroxaban with or without aspirin in stable cardiovascular disease. N Engl J Med 2017;377(14):1319-1330

52 ClinicalTrials.gov. NCT01776424. Rivaroxaban for the prevention of major cardiovascular events in coronary or peripheral artery disease (COMPASS). Available at: https://ClinicalTrials.gov/show/ NCT01776424. Accessed February 21, 2018

53 Bosch J, Eikelboom JW, Connolly SJ, et al. Rationale, design and baseline characteristics of participants in the Cardiovascular Outcomes for People Using Anticoagulation Strategies (COMPASS) Trial. Can J Cardiol 2017;33(08):1027-1035

54 Anand S; on Behalf of COMPASS Investigators. Rivaroxaban in stable peripheral or carotid artery disease. Presented at the European Society of Cardiology Congress 2017, Barcelona, Spain; 2017

55 Fox KF, Cowie MR, Wood DA, et al. Coronary artery disease as the cause of incident heart failure in the population. Eur Heart J 2001; 22(03):228-236

56 Gheorghiade M, Sopko G, De Luca L, et al. Navigating the crossroads of coronary artery disease and heart failure. Circulation 2006;114(11):1202-1213

57 Cugno M, Mari D, Meroni PL, et al. Haemostatic and inflammatory biomarkers in advanced chronic heart failure: role of oral anticoagulants and successful heart transplantation. $\mathrm{Br} \mathrm{J}$ Haematol 2004;126(01):85-92 
58 Gibbs CR, Blann AD, Watson RD, Lip GY. Abnormalities of hemorheological, endothelial, and platelet function in patients with chronic heart failure in sinus rhythm: effects of angiotensinconverting enzyme inhibitor and beta-blocker therapy. Circulation 2001;103(13):1746-1751

59 Watson RD, Gibbs CR, Lip GY. ABC of heart failure. Clinical features and complications. BMJ 2000;320(7229):236-239

60 McMurray JJ, Adamopoulos S, Anker SD, et al; ESC Committee for Practice Guidelines. ESC Guidelines for the diagnosis and treatment of acute and chronic heart failure 2012: The Task Force for the Diagnosis and Treatment of Acute and Chronic Heart Failure 2012 of the European Society of Cardiology. Developed in collaboration with the Heart Failure Association (HFA) of the ESC. Eur Heart J 2012;33(14):1787-1847

61 Homma S, Thompson JL, Pullicino PM, et al; WARCEF Investigators. Warfarin and aspirin in patients with heart failure and sinus rhythm. N Engl J Med 2012;366(20):1859-1869

62 Cleland JG, Findlay I, Jafri S, et al. The Warfarin/Aspirin Study in Heart failure (WASH): a randomized trial comparing antithrombotic strategies for patients with heart failure. Am Heart J 2004; 148(01):157-164

63 Cokkinos DV, Haralabopoulos GC, Kostis JB, Toutouzas PK; HELAS Investigators. Efficacy of antithrombotic therapy in chronic heart failure: the HELAS study. Eur J Heart Fail 2006;8(04):428-432

64 Lip GY, Shantsila E. Anticoagulation versus placebo for heart failure in sinus rhythm. Cochrane Database Syst Rev 2014;(03): CD003336

65 Glasser SP, Salas M, Delzell E. Importance and challenges of studying marketed drugs: what is a phase IV study? Common clinical research designs, registries, and self-reporting systems. J Clin Pharmacol 2007;47(09):1074-1086

66 A Study Exploring Two Strategies of Rivaroxaban (JNJ39039039; BAY-59-7939) and One of Oral Vitamin K Antagonist in Patients With Atrial Fibrillation Who Undergo Percutaneous Coronary Intervention (PIONEER AF-PCI). Available at: http://clinicaltrialsgov/show/NCT018305432014. Accessed February 21, 2018

67 Zannad F, Greenberg B, Cleland JG, et al. Rationale and design of a randomized, double-blind, event-driven, multicentre study comparing the efficacy and safety of oral rivaroxaban with placebo for reducing the risk of death, myocardial infarction or stroke in subjects with heart failure and significant coronary artery disease following an exacerbation of heart failure: the COMMANDER HF trial. Eur J Heart Fail 2015;17(07):735-742

68 ClinicalTrials.gov. NCT01877915. A study to assess the effectiveness and safety of rivaroxaban in reducing the risk of death, myocardial infarction or stroke in participants with heart failure and coronary artery disease following an episode of decompensated heart failure (COMMANDER HF). Available at: https://ClinicalTrials.gov/show/ NCT01877915. Accessed February 21, 2018

69 Pocock SJ, Elbourne DR. Randomized trials or observational tribulations? N Engl J Med 2000;342(25):1907-1909

70 Ohman EM, Roe MT, Steg PG, et al. Clinically significant bleeding with low-dose rivaroxaban versus aspirin, in addition to P2Y12 inhibition, in acute coronary syndromes (GEMINI-ACS-1): a doubleblind, multicentre, randomised trial. Lancet 2017;389(10081): 1799-1808

71 Angiolillo DJ, Capodanno D, Goto S. Platelet thrombin receptor antagonism and atherothrombosis. Eur Heart J 2010;31(01):17-28 